\title{
Forgotten exogenous corticosteroid as a cause of central serous chorioretinopathy
}

\author{
Paul W Hardwig' \\ Amila O Silva ${ }^{2}$ \\ Jose S Pulido' \\ 'Department of Ophthalmology, Mayo \\ Clinic, Rochester, MN, USA; ${ }^{2}$ Kaiser \\ Permanente Fontana Medical Center, \\ Fontana, CA, USA
}

\begin{abstract}
Central serous chorioretinopathy (CSCR) is an idiopathic ocular condition - first described in 1866 - that is well known to ophthalmologists. It is less well known to other practitioners. Glucocorticoids have been strongly implicated as a pathogenic factor. We report three patients who developed CSCR following exogenous administration of corticosteroid. Because our patients did not suspect the use of corticosteroid to be important or causative, they did not volunteer the historical detail, and admitted to exogenous corticosteroid injection only with intensive questioning. For their part, physicians should be cognizant of the risk of corticosteroidinduced CSCR, particularly in patients with a prior history of the potentially sight-threatening disease. The development of CSCR is an important iatrogenic and often unrecognized side effect of exogenously administered corticosteroid.
\end{abstract}

Keywords: exogenous corticosteroid, ocular complication, central serous chorioretinopathy

CSCR is an idiopathic ocular condition characterized by pathologic accumulation of fluid at the posterior pole of the fundus, creating a localized retinal detachment (Klais et al 2006). Patients present with decreased vision, metamorphopsia and/or chromatopsia when the macular retina is involved, but are generally asymptomatic with extrafoveal, eccentric lesions. The disease typically affects young and middleaged adults, with men more commonly affected than women. Most cases resolve spontaneously with visual recovery, but recurrences are common and may lead to permanent visual loss.

The pathogenesis of the disease, although incompletely understood, has been attributed to dysfunction in the retinal pigment epithelium (RPE) (Spitznas 1986), the choroid (Piccolino and Borgia 1994), or both. Glucocorticoids have been strongly implicated as a pathogenic factor, since CSCR occurs both in conditions of endogenous hypercortisolism such as Cushing's disease and in the setting of exogenously administered glucocorticoids (Bouzas et al 2002). CSCR may develop when exogenous corticosteroid is administered by any number of routes - including systemic (oral, intravenous), local (intramuscular, intraarticular, epidural) and topical (inhalational, intranasal, and dermatologic; Karadimas et al 2004). Accordingly, some have argued that CSCR should be added to the list of ocular complications of glucocorticoids, administered by any route (Bouzas et al 2002; Karadimas and Bouzas 2004).

We report three patients who developed CSCR with exogenous administration of steroid. Of particular note, all 3 patients initially denied recent corticosteroid use. This retrospective study was approved by the Institutional Review Board of Mayo Foundation, and was Health Insurance Portability and Accountability Act-compliant. 


\section{Brief report of cases Case I}

A 67-year-old male presented with a four-week history of blurred vision in the left eye. The patient initially presented to his primary-care physician who attributed the visual decline to a cataract. Past medical history was significant for adequately controlled hypertension.

On referral to ophthalmology, the patient denied any form of corticosteroid use. After intensive questioning, he admitted to having an intramuscular corticosteroid injection for shoulder pain 6 weeks prior to the visit.

Best corrected visual acuity was 20/20 right eye and 20/60 left eye. On funduscopy, there were subtle RPE changes in both maculas, and subretinal fluid in the left eye temporal to the fovea. A fluorescein angiogram (FA) demonstrated an irregular patch of progressive hyperfluorescence within the area of subretinal fluid in the left eye, consistent with CSCR.

The patient was initially observed. At 10-day follow-up, vision in the left eye had decreased to 20/200. Laser photocoagulation was performed. Ten days post-treatment, vision had improved to $20 / 50$, and subretinal fluid had decreased on funduscopic exam.

\section{Case 2}

A 40-year-old normotensive male related a two-day history of a central shadow in his right eye. Past eye history was remarkable for a previous episode of CSCR in the right eye that spontaneously resolved 18 months before the current episode. Medical history was significant for seasonal allergies. He initially denied corticosteroid use, but subsequently remembered receiving a corticosteroid injection for an exacerbation of allergies one month prior to presentation.

Best corrected visual acuity was 20/20 in both eyes. On dilated funduscopy, there was a 1.5 disc-diameter area of subretinal fluid in the right eye extending from the superior edge of the fovea. On FA, there was a pinpoint area of progressive hyperfluorescence within the region of subretinal fluid. A diagnosis of recurrent CSCR was made. No treatment was advised, given the presenting vision.

\section{Case 3}

A 72-year-old male presented with a complicated eye history. The patient was pseudophakic in both eyes. He had 2 prior episodes of CSCR in the left eye - in 1992 and again in 1999 - both of which remitted spontaneously without visual compromise. In January 2006, a rhegmatogenous retinal detachment and a large choroidal melanoma were diagnosed in the right eye. The melanoma was treated elsewhere with brachytherapy in March 2006.

The patient presented to our institution in May 2006 for continuing followup of the melanoma in his right eye. There were no visual concerns in his left eye. Past medical history was significant for treated hypertension and thyroidreplacement therapy. Only on intensive questioning did the patient admit to having two recent epidural corticosteroid injections for low back pain, 4 to 7 weeks prior to his Mayo Clinic visit.

On presentation, visual acuity was count fingers right eye and 20/20 left eye. There were well positioned posterior chamber intraocular lenses in both eyes. In the right eye, there was a large ciliochoroidal melanoma, and a fibrovascular and total retinal detachment. In the fellow eye, there were fine pigment changes under the fovea. An optical coherence tomography of the left macula was obtained: the paramacular neurosensory retina was elevated, consistent with active CSCR. No treatment was undertaken, given the asymptomatic nature of the lesion.

\section{Discussion}

Of a number of risk factors that predispose to the development of CSCR, glucocorticoids have been strongly implicated as a pathogenic factor (Haimovici et al 2004). The prevalence of exogenous administration of glucocorticoid in patients developing CSCR has been reported at less than 10 percent in 3 large retrospective studies (Lowder et al 1981; Wakakura et al 1987; Tittl et al 1999). In 2 recent prospective studies, prevalence was reported at $29 \%$ and $52 \%$, and in each study was statistically significant compared with casematched controls (Carvalho-Recchia et al 2002; Karadimas and Bouzas 2004). As reviewed by Bouzas and colleagues (2002), CSCR may develop from a few days to several years after the initiation of exogenous corticosteroid, and in doses as low as $10-15 \mathrm{mg}$ orally per day. The elderly population is particularly vulnerable to small amounts of corticosteroid (Koyama et al 2004).

Primary-care providers who routinely administer exogenous corticosteroid should be aware of the glucocorticoids - CSCR connection. Case 1 highlights that accurate diagnosis may be elusive without an index of suspicion. Providers should be mindful that CSCR may develop or recur with exogenous corticosteroid administered at low doses and by any route.

Our report underscores the need for careful history taking on the part of both primary- and eye-care providers. Primary care should elicit a careful eye history before the 
administration of exogenous corticosteroid. In particular, inquiries of a prior history of CSCR or episodes of blurred central vision should be made. Cases 2 and 3 demonstrate that patients with prior disease may be given exogenous corticosteroid unwittingly, and may then go on to develop recurrent CSCR. For the ophthalmologist's part, queries of a prior history of corticosteroid use in any form should be made when a patient presents with CSCR. Since patients may not view one-time or pulsed administration - such as an intramuscular injection - as corticosteroid use worth reporting or in any way related to their current eye problem, they may fail to mention it during medication inventory. All three case reports demonstrate that patients with CSCR may not readily volunteer information of recent corticosteroid use. To more fully capture corticosteroid use, the ophthalmologist should review the past medical history, inquiring specifically about conditions that are often treated with corticosteroid (eg, allergies, asthma, joint pain). Without a complete log of recent medications, exogenous corticosteroid may be neglected, leading to a general underreporting of corticosteroid-induced cases of CSCR.

Finally, a heightened awareness of the corticosteroid CSCR connection on the part of the primary provider and the patient will insure a more fully informed consent. Aware of the risk of new or recurrent CSCR, the provider should convey that either event - but particularly recurrent disease - can lead to permanent visual loss.

In summary, this report reinforces CSCR as a potential ocular complication of corticosteroid therapy in any form. It emphasizes that patients may not readily volunteer corticosteroid use - especially when given a single dose in a form such as intramuscular injection - and underscores the need for a careful history and inventory of recent medications. Finally, it serves to heighten awareness of primary-care providers to this potentially sight-threatening ocular complication.

\section{Disclosure}

Supported in part by Research to Prevent Blindness, Inc, New York, New York, and by Mayo Foundation, Rochester, Minnesota. There is no conflict of interest.

\section{References}

Bouzas EA, Karadimas P, Pournaras CJ. 2002. Central serous chorioretinopathy and glucocorticoids. Surv Ophthalmol, 47:431-48.

Carvalho-Recchia CA, Yannuzzi LA, Negrao S, et al. 2002. Corticosteroids and central serous chorioretinopathy. Ophthalmology, 109:1834-7.

Haimovici R, Koh S, Gagnon DR, et al. 2004. Risk factors for central serous chorioretinopathy: a case-control study. Ophthalmology, 111:244-9.
Karadimas P, Bouzas EA. 2004. Glucocorticoid use represents a risk factor for central serous chorioretinopathy: a prospective, case-control study. Graefes Arch Clin Exp Ophthalmol, 242:800-2.

Karadimas P, Kapetanios A, Bouzas EA. 2004. Central serous chorioretinopathy after local application of glucocorticoids for skin disorders. Arch Ophthalmol, 122:784-6.

Klais CM, Ober MD, Ciardella AP, et al. 2006. Central Serous Chorioretinopathy. In: Ryan SJ, Schachat AP (eds). Retina. vol 2. 4th ed. Philadelphia: Mosby, pp. 1135-61.

Koyama M, Mizota, A, Igarashi Y, et al. 2004. Seventeen cases of central serous chorioretinopathy associated with systemic corticosteroid therapy. Ophthalmologica, 218:107-10.

Lowder CY, Gutman FA, Zegarra H, et al. 1981. Macular and paramacular detachment of the neurosensory retina associated with systemic diseases. Trans Am Ophthalmol Soc, 79:347-70.

Piccolino FC, Borgia L. 1994. Central serous chorioretinopathy and indocyanine green angiography. Retina, 14:231-42.

Spitznas M. 1986. Pathogenesis of central serous retinopathy: a new working hypothesis. Graefes Arch Clin Exp Ophthalmol, 224:321-4.

Tittl MK, Spaide RF, Wong D, et al. 1999. Systemic findings associated with central serous chorioretinopathy. Am J Ophthalmol, 128:63-8.

Wakakura M, Song E, Ishikawa S. 1987. Corticosteroid-induced central serous chorioretinopathy. Jpn J Ophthalmol, 41:180-5. 
